\title{
Anisotropic magnetic field-effect on martensitic transformation for Co and Co alloys
}

\author{
T. Tanaka, M. Takahashi ${ }^{1}$ and S. Kadowaki ${ }^{1}$ \\ Department of Materials Science and Engineering, Ehime University, Matsuyama 790-8577, \\ Japan \\ ${ }^{1}$ Department of Electronic Engineering, Tohoku University, Sendai 980-8579, Japan
}

\begin{abstract}
The experimental evidences on the anisotropic field-induced martensitic transformation were summarized for the cases of the fcc-to-hcp, fcc-to-dhcp and dhcp-to-hcp martensitic trasformation in Co and Co alloys. In these phase transformations, the magnitude of the magnetic field as small as some kilo Oe was enough to induce the martensitic transformation and the magnetic field acted anisotropically. The magnetocrystalline anisotropy energy was explained as the main motive force of this anisotropic field-induced martensitic transformation from the thermo-dynamical consideration. The shear stresses regarding to the magnetostriction are estimated to be the same order of the reported value of the resolved shear stress for $\mathrm{Co}$, and these shear stresses did not meet the experimental results concerning to the direction of the external field clearly. The shear stress together with the magnetostriction would play an important role on the formation of the nucleation site of the martensitic tansformation in the domain walls.
\end{abstract}

\section{INTRODUCTION}

Recently, the development of the high field techniques supported by superconducting magnet technology has opened the new world of experimental research under magnetic fields as strong as same ten $T$. Fascinating works concerning to the diffusion in the solids, the structure transformation and the grain growth have been started [1-3]. The effects of the external magnetic field are observed remarkably under a strong magnetic field. The role of the magnetic field is interesting to investigate and are now under study.

Pioneer works concerning to the effect of the magnetic field on the martensitic transformation in steels were investigated by the Russian scientists using a normal electro-magnet technique in the $60 \mathrm{~s}$ [4-6]. The martensite phase in steels were induced by the external magnetic field under the field generated by a normal electro-magnet. Further investigation was performed under the strong magnetic field generated by a pulse magnetic field [7]. This field-induced martensitic phase transformation was mainly explained by the magnetostatic free energy difference between the ferromagnetic martensitic phase and non-magnetic mother phase. The features of this field-induced martensitic transformation are that the external magnetic field acts isotropically on the phase transformation and that the martensite-start (Ms) temperature rises proportionally to the field strength. This type of field induced martensitic transformation is more clearly observed in a strong magnetic field.

We found another type of field-induced martensitic transformation in Co and Co alloys. Cobalt is the only ferromagnetic metal that has a martensitic phase transformation from a face-centered cubic (fcc) structure to a hexagonal-closed packed (hcp) structure in its ferromagnetic phase. The magnetization difference between those phases is reported to be less than $1.5 \%$ [8] at the phase transition temperature. As the magnetic energy was negligibly small when compared to the chemical energy for the martensitic transformation, any experimental and theoretical investigation has not considered the effect of the external magnetic field on this martensitic transformation in Co for a long time. Recently we found the experimental evidence, which clearly supported the existence of the field-induced martensitic transformation in $\mathrm{Co}$ and $\mathrm{Co}-\mathrm{Ni}$ alloys [9]. The difference from the case of steels that is an external magnetic field as small as some kilo Oe is enough to induce the martensitic phase transformation and the magnetic field affects on it anisotropically [10]. We also found that the double hexagonal-closed 
packed (dhcp) structure transform into the hcp structure by the application of magnetic field for Co-Fe alloys near room temperature [11]. This field-induced martensitic transformation is also anisotropic.

In this paper, we will summarize our experimental results concerning the anisotropic field-induced martensitic transformation for $\mathrm{Co}$ and $\mathrm{Co}$ alloys and discuss the magnetic field-effect on the martensitic transformation in these metal and alloys.

\section{EXPERIMENTAL EVIDENCES}

\subsection{Fec-to-hep martensitic transformations}

The field-effect on the fcc-to-hep martensitic transformation was found in $\mathrm{Co}$ and $\mathrm{Co}-\mathrm{Ni}$ alloys, through the investigation of the mechanism of the field-induced magnetic anisotropy. It was reported that the magnetic anisotropy was induced in polycrystalline hexagonal $\mathrm{Co}$ and $\mathrm{Co}-\mathrm{Ni}$ alloys when they were cooled under the external magnetic field. The mechanism was not understood [12]. We investigated the mechanism from the point of the formation of a preferred orientation of hcp grains by the external magnetic field.

The disk shaped specimens of polycrystalline $\mathrm{Co}$ and $\mathrm{Co}-\mathrm{Ni}$ alloys were cooled from $1000^{\circ} \mathrm{C}$ to room temperature under an external magnetic field, $H_{\mathrm{ex}}=10 \mathrm{kOe}$. The hcp grain orientation was detected by the standard X-ray Schultz's reflection and transmission technique, and was compared to the hcp grain orientation of the samples, which were cooled without magnetic field. The induced magnetic anisotropy was determined from the torque measurement.

We succeeded to detect the preferred orientation of hop grains that was formed by cooling in the external magnetic field and to confirm that the preferred orientation was the origin of the induced magnetic anisotropy. The (0002) intensities for sample cooled in the magnetic field do not show a random distribution against the azimuth angle $\beta$ but a periodic one. This periodicity is not observed in the samples cooled without the magnetic field. Strong intensities are observed around $\beta=90^{\circ}$ and $270^{\circ}$ for Co. Here $\beta=90^{\circ}$ corresponds to the perpendicular direction to the external magnetic field. A pole figure of the hcp (0002) grains is shown in Fig.1. This pole figure suggests that the c-axis of Co turns to the direction perpendicular to the magnetic field. The magnitude of the induced magnetic anisotropy obtained by the torque method agreed well with the calculated value based on the preferred orientation and the magnetocrystalline anisotropy constant.

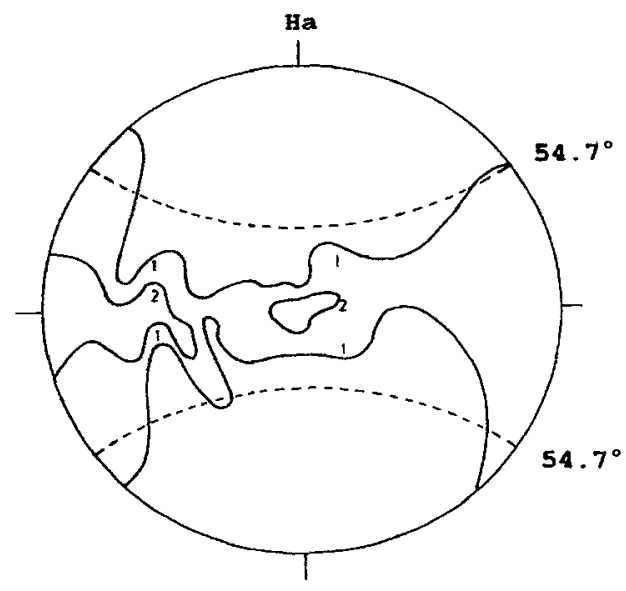

Figure 1 The (0002) pole figure for the magnetically annealed $\mathrm{Co}$. Ha indicates the field direction during the cooling. The high-density pole area is found to be perpendicular to the field direction. This corresponds to the easy direction of magnetocrystalline anisotropy at the phase transition temperature. 
Assuming that the magnetic field affects anisotropically the fcc-to-hcp martensitic transformation, the formation of the preferred orientation is easily understood. We will discuss the anisotropic field-induced effect on the martensitic transformation in Chapter 3.

\subsection{Fcc-to-dhcp martensitic transformation}

The dhcp phase appears in Co-Fe alloys at the iron concentration from 1 to $5 \%$. In this concentration range, the high temperature fcc phase transforms to the low temperature dhcp phase. This martensitic transformation has also the possibility to be affected by the magnetic field as the same condition as the fcc-to-hcp structure change occurs. We investigated this possibility by detecting the magnetic anisotropy induced by the cooling in magnetic field for Co-Fe-Ni ternary alloys.

The polycrystalline disk specimens for Co-rich $\mathrm{Co}-\mathrm{Fe}-\mathrm{Ni}$ alloys were prepared by the casting and forging method to make a randomly oriented grain. The samples are cooled from $1000^{\circ} \mathrm{C}$ in a $10 \mathrm{k} \mathrm{Oe}$ field. The induced magnetic anisotropy was determined by the torque method. Figure 2 shows the magnitude of the induced magnetic anisotropy. All the samples examined were found to be affected by the external magnetic field and the magnetic anisotropy was found to be induced by cooling in a magnetic field. The positive and negative sign mean that the induced easy direction is parallel or perpendicular to the field direction. Furthermore, they correspond to the fcc-to-dhcp or fcc-to-hcp martensitic transformation, respectively. Consequently, the fcc-to-dhcp martensitic transformation was found to be affected by the external magnetic field, too.

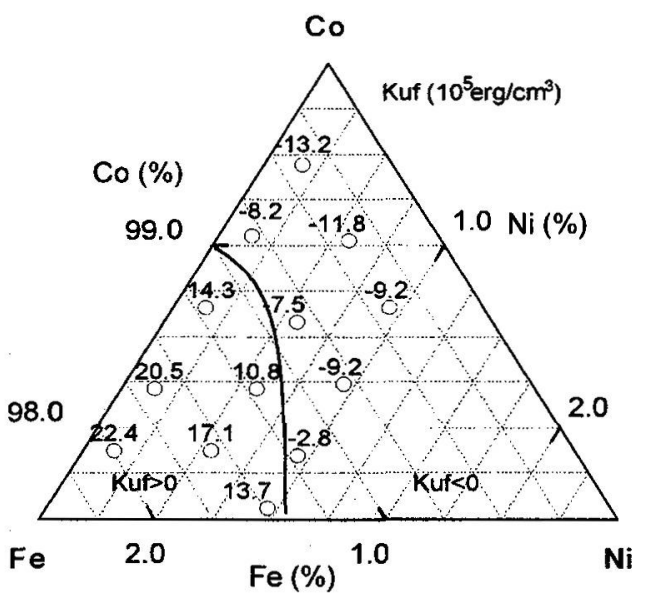

Figure 2 The induced magnetic anisotropy Kuf for Co-rich Co-Fe-Ni alloys. The positive sign area corresponds to the fcc-dhcp martensitic transformation and the negative sign indicates the fcc-to-hcp martensitic transformation.

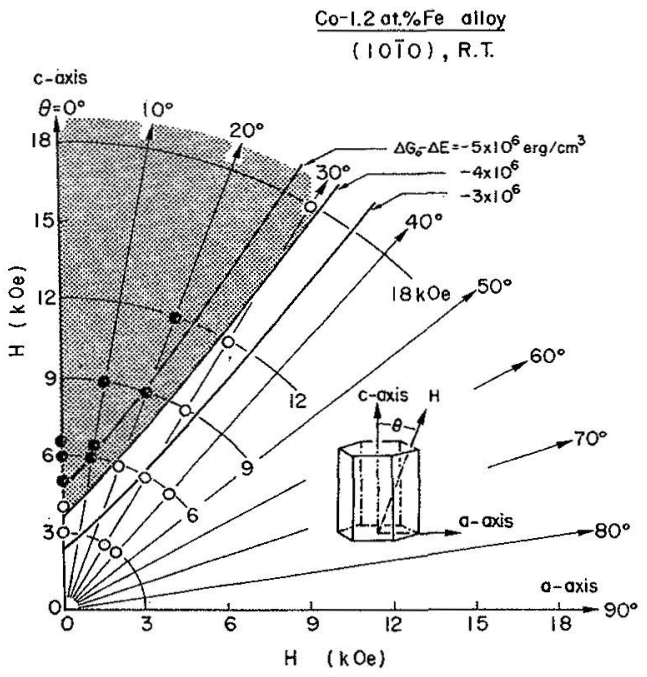

Figure 3 The magnetic field direction and strength required for the field-induced dhcp-to-hcp martensitic transformation. The shaded area shows that the martenistic transformation is induced by the magnetic field at room temperature. 


\subsection{Dhcp-to-hcp martensitic trnsformation}

One more example for the direct evidence on the anisotropic field-induced martensitic transformation was found in dhcp-to-hcp martensitic transformation, as observed in the Co-Fe alloy single crystals. The Co-Fe alloys for iron concentration between 0.8 to $1.2 \%$ show the dhcp-to-hcp martensitic transformation subsequent to the fcc-to-dhcp martensitic transformation. Especially in the $\mathrm{Co}-1.2 \% \mathrm{Fe}$ alloy, the dhcp structure is stable at room temperature and the dhcp-to-hcp martensitic transformation takes place only when the field is applied. This field-induced martensitic transformation depends strongly on the field direction and strength. Figure 3 shows the direction and the magnitude of the field for starting the field induced martensitic transformation. The minimum field strength required to start the field induced martensitic transformation is $4 \mathrm{k}$ Oe when the field was applied parallel to the c-axis. The magnitude increased when the angle between field and $c$-axis was increased. Finally, magnetic field of $18 \mathrm{kOe}$ is requested when the field was applied $30^{\circ}$ from the c-axis. This boundary depends on the temperature.

\section{EFFETC OF THE MAGNETIC FIELD}

As mentioned above, the magnetic field acts anisotropically on the martensitic transformation in Co and Co alloys. This point is the most important characteristics, which is different from the many kinds of steels. Here, we will discuss the effects of the anisotropic magnetic field-effect on the martesitic transformation.

\subsection{Role of the magnetocrystalline anisotropy energy}

The magnetostatic energy originated from the scalar product of magnetization and magnetic field is the main source of many kinds of field-effects in solids. The field-induced martensitic transformation in steels is also understood by considering the free energy difference between non-ferromagnetic (parent) phase and ferromagnetic martensite. On the contrary, magnetocrystalline anisotropy energy should play an important role in the present anisotropic field-effect. Here, we will discuss the role of the magnetocrystalline anisotropy energy as a motive force of the martensitic transformation from the thermodynamic point of view.

The free energy $G$ is expressed by the sum of a lattice term $G_{0}$ and a magnetic term $G_{\text {mag. }}$. The lattice term consists of an internal energy term $U_{\theta}$ and an entropy term $S T$, where $S$ is entropy and $T$ is temperature, and the magnetic term consists of a magnetic anisotropy energy $E_{a}$, Zeeman energy $E_{z}$ and magnetoelastic energy $E_{e l}$. For the present fcc-hcp, fcc-dhcp or dhcp-hcp martensitic transformations, the magnetoelastic enery is estimated to be as small as $10^{3} \mathrm{erg} / \mathrm{cm}^{3}$ assuming that the magnitude of the internal strain is the same order of its magnetostriction. Consequently, the free energy $G$ is expressed as a function of temperature and field by,

$$
G(T, H)=\left(U_{0}+T S\right)+\left(E_{a}+E_{z}\right) .
$$

Thus, the free energy difference between the parent phase and martensitic phase is

$$
\Delta G(T, H)=\Delta G_{0}+\Delta G_{m} .
$$

The martensitic transformation will progress when the free energy difference will exceed the potential barrier $\Delta E$ as following the criterion that was applied to understand the stress induced martensitic transformation by.Patel and Cohen [13].

We calculated this free energy difference of the magnetic part $\Delta G_{m}$ for the fcc-to-hcp and dhcp-to-hcp martensitic transformation using the reported value of magnetization and magnetocrystalline anisotropy energy $[10,11]$. In Figure 4 , an example of the free energy difference for the fcc-hcp transformation of $\mathrm{Co}(110)$ plain is indicated as a function of the field strength. The free energy differences are clearly indicated and strongly depend on the field direction $\phi$. Assuming the sum 
of this anisotropic free energy difference and the isotropic lattice energy term can exceed the potential barrier, the formation of the preferred orientation of hcp grains is understood easily.

The calculated results for the case of dhcp-hcp martensitic transformation are also indicated in Fig. 3 as a function of the $\Delta G_{\sigma^{-}} \Delta E$ for Co-1.2\%Fe alloy. When we choose $\Delta G_{\theta^{-}} \Delta E$ is $-4 \times 10^{6} \mathrm{erg} / \mathrm{cm}^{3}$, the calculated values are in good agreement with the experimental results. It means that the additional energy of $4 \times 10^{6} \mathrm{erg} / \mathrm{cm}^{3}$, which is needed to exceed the energy barrier $\Delta E$, is supplied by the magnetic energy, by the anisotropy energy and Zeeman energy. These results strongly suggested that the magnetocrystalline anisotropy energy induces the martensitic transformation for $\mathrm{Co}$ and $\mathrm{Co}$ alloys.

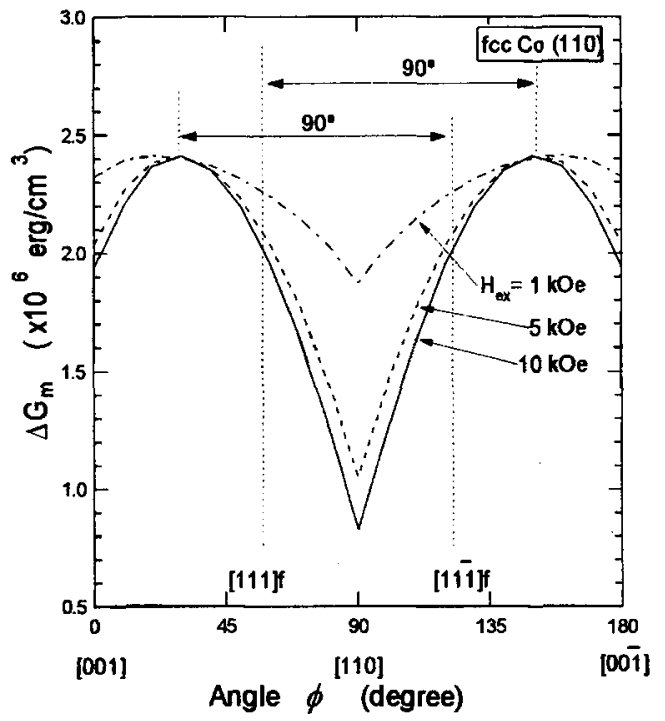

Figure 4 The magnetic energy difference $\Delta G_{m}$ for $\operatorname{Co}(110)$ as a function of applied field. The $\Delta G_{m}$ is strongly depends on the angle $\phi$ which is the angle between the magnetic field and [001] direction.

\subsection{Magnetostriction}

From the thermodynamical point of view, the magnetocrystalline anisotropy energy is understood as a main energy source for the anisotropic field-effect for the martensitic transformation. The shear stress in the crystal is also essential in the martensitic transformation from the kinetic point of view. The ferromagnetic materials deform their shape by magnetostriction when they are magnetized. Occurrence of this deformation, the shear stresses are generated in their body. These shear stresses are strongly connected with the magnetostriction constant and external magnetic field. We also have to consider the effect of the shear stresses, which are varied by the external magnetic field. The effect of the magnetostriction was considered on dhcp-hcp field induced martensitic transformation.

The shear stresses are calculated using the magnetostriction constants and the elastic stiffness constants. For the case of Co- $1.2 \% \mathrm{Fe}$, the shear stresses $\sigma_{13}$ and $\sigma_{23}$ in the crystal can be expressed by the following relation,

$$
\begin{aligned}
& \sigma_{13}=-\frac{1}{2}\left(\lambda_{A}+\lambda_{c}-4 \lambda_{D}\right) C_{44} \sin \Psi, \\
& \sigma_{23}=-\frac{1}{2}\left(\lambda_{A}+\lambda_{c}-4 \lambda_{D}\right) C_{44} \sin \Psi,
\end{aligned}
$$


where $\lambda_{A}, \lambda_{B}, \lambda_{C}$ and $\lambda_{D}$ are the magnetostriction constants and $\Psi$ is the angle between the c-axis and magnetization.

We calculate the magnitude of the $\sigma_{13}$ using the magnetostriction constants $\lambda_{\mathrm{A}}=-6.5 \times 10^{-6}$, $\lambda_{C}=55 \times 10^{-6}, \lambda_{D}=-12.9 \times 10^{-6}$ for $\mathrm{Co}-1.2 \% \mathrm{Fe}$ alloy at room temperature [14] and $C_{44}=0.755 \times 10^{12} \mathrm{dyn} / \mathrm{cm}^{2}$ for $\mathrm{Co}[15]$ instead of Co-Fe alloys. The elastic constants for $\mathrm{Co}-\mathrm{Fe}$ alloys are not reported at present. The calculated value of $-\frac{1}{2}\left(\lambda_{A}+\lambda_{C}-4 \lambda_{D}\right) C_{44}$ is $-3.8 \times 10^{7} \mathrm{dyn} / \mathrm{cm}^{2}$. The magnitude of the resolved shear stress for Co is reported to be $7 \times 10^{7} \mathrm{dyn} / \mathrm{cm}^{2}$ [16]. Thus, this shear stress is nearly the same value of the calculated one. The maximum shear stress, however, is obtained when the magnetization turns to an angle of $45^{\circ}$ from the c-axis. No shear stress is generated when the magnetization turns to be parallel to the c-axis. This situation does not meet the experimental result as shown in Fig.3. Consequently, magnetostriction in this domain has no effect on the anisotropic field-effect. On the other hand, the magnetization in the magnetic domain wall changes its direction from one magnetic domain to the other. During this transition layer, the magnetization direction passes through the angle $45^{\circ}$ from the c-axis. Thus, the resolved shear stress might be applied in the magnetic domain walls. This would play an important role for the nucleation of the martesitic phase. The domain walls might be one of the nucleation sites.

From these arguments, we can conclude the following mechanism of the anisotropic field-effect on the martensitic transformation. Magnetocrystalline anisotropy energy is found to be the main activation energy for the anisotropic field-induced transformation. Magnetostriction in the domain walls would play an important role to generate the nucleation sites of the martensitic phase.

\section{Acknowledgement}

The authors are particularly indebted to Professor T.Kakeshita at Osaka University for many suggestions on the martensitic transformation.

\section{References}

1. R.Ohshima, H.Tokoro, J.Jpn.Inst.Metals 35, 317 (1998).

2. Y.Belli, R.K.Mishra, Mater. Sci. Eng. 47, 69 (1981).

3. T.Watanabe, Y.Suzuki, S.Tanii, H.Oikawa, phil.Mag.Lett. 62, 9 (1990).

4. V.D.Sadovskii, N.M.Rodigin, L.V.Smimov, G.M.Filonchik, I.G.Fakidov, Fiz.Met.Metalloved. 12, 302 (1961).

5. Ye.A.Fokina, E.A.Zavadoskiy, Fiz.Met.Metalloved. 16, 311 (1963).

6. E.I.Estrin, Fiz.metal.metalloved. 19, 929 (1965).

7. T.Kakeshita, K.Shimizu, S.Funada, M.Date, Acta Metall. 33, 1381 (1985)

8. H.P.Meyers, W.Sucksmith, Proc.R.Soc.London, Ser.A 207, 427 (1951).

9. T.Tanaka, M.Takahashi, S.Kadowaki, J.Appl.Phys. 84, 6768 (1998).

10.T.Tanaka, M.Takahashi , S.Kadowaki, Proceedings of the Int. Conf. Solid-Solid Phase Transformations '99, 977 (1999).

11. T.Tanaka, M.Takahashi, S.Kadowaki, Phys.Rev. B60, 12256(1999).

12. M.Takahashi,T.Kono, J.Phys.Soc.Jpn. 15, 936 (1960).

13. J.R.Patel, M.Cohen, Acta Metall. 1, 532 (1953).

14. S.Ishio, M.Takahashi, J.Magn.Magn.Mater. 46, 142 (1984).

15. H.J.McSkimin, J.Appl.Phys. 26, 406 (1955).

16. R.T.Holt, Cobalt 56, 145 (1972). 